\title{
Transient excitonic states in noble metals and Al
}

\author{
Wolf-Dieter Schöne and Walter Ekardt \\ Fritz-Haber-Institut der Max-Planck-Gesellschaft, Faradayweg 4-6, D-14195 Berlin, Germany \\ (Received 25 July 2001; revised manuscript received 4 December 2001; published 8 March 2002)
}

\begin{abstract}
Bound states between electrons and holes, so-called excitons, are an ubiquitous feature in the spectra of excited states of molecules, clusters, and in a variety of solids such as semiconductors or ionic solids. It is common wisdom that excitons do not exist in metals because the attractive, long-range particle-hole interaction which is needed for the formation of an exciton does not exist in metals due to almost complete screening. We showed in a previous publication that excitons in metals can exist as transient states right after the creation of the electron-hole pair which explained in a natural way the up to then not understood transitions seen in $\mathrm{Cu}$ in the experimental spectra of time-resolved two-photon photoemission spectroscopy. In this paper we report about transient excitonic states in Ag and Au. We explain why these states have not yet been detected in Ag but we predict their existence. We show furthermore why transient excitonic states cannot be built up in Al.
\end{abstract}

DOI: 10.1103/PhysRevB.65.113112

PACS number(s): 78.47.+p, 72.15.Lh, 71.10.-w, 71.20.Gj

With the advent of modern femtosecond laser technology it has become possible to measure ultrafast relaxation processes of excited electrons in metals. ${ }^{1-3}$ Whereas in most cases the experimentally observed states can be identified as single-particle band states there are other cases where the experimental results cannot be explained on the basis of a single-particle band structure. Most prominent are the measurements of the lifetime of excited electrons using timeresolved two-photon photoemission (TR-2PPE) spectroscopy performed on single crystals of $\mathrm{Cu}$ (Refs. 1,2,4) and Au. ${ }^{5}$ They show data for states in the (100) and (111) direction in an energy range between 1 and $4 \mathrm{eV}$. We are not aware of any such data for Ag. These two directions are of special interest since the band structures for all three noble metals do not show single-particle states in these directions for the energy range under consideration $(1-4 \mathrm{eV}) .{ }^{6}$ There is a second observation; Ogawa et al. ${ }^{1}$ also measured the lifetime of excited electrons in the $\mathrm{Cu}(110)$ direction. Since this direction shows a single-electron band the lifetime of electrons in this band has been calculated using a state-of-the-art $a b$ initio many-body calculation within the $G W$ approximation. A comparison between the theoretical and experimental data reveals good agreement for band energies above $2 \mathrm{eV}$ but it shows a huge discrepancy for energies below $2 \mathrm{eV}^{7,8}$ Alike results have been found by similar calculations. ${ }^{9}$ This shows that the $G W$ approximation does not contain enough physics to explain the lifetime of hot electrons in $\mathrm{Cu}$. This is noteworthy because calculations utilizing the $G W$ approximation typically lead to very good agreement with experimental data, a fact that laid the foundation for its big success. ${ }^{10}$ It was speculated that the observed states are caused by an attractive interaction between the photoelectron and its localized hole in the $d$ bands. ${ }^{4}$ In a recent paper ${ }^{11}$ we presented a model which substantiated this idea.

The basic principle on which our model is based on is the fact that screening in an electronic system is a dynamic process and that it therefore always takes an electronic system a short but finite time to react to any perturbation. This is reflected by the frequency dependence of the dielectric function $^{12} \epsilon\left(\mathbf{r}, \mathbf{r}^{\prime} ; \omega\right)$ or by the time dependence of its Fourier transform $\epsilon\left(\mathbf{r}, \mathbf{r}^{\prime} ; t\right)$, respectively. The basic assumption behind our model is that the first pulse in a TR-2PPE experiment-the so-called pump pulse - creates an electronhole pair. Immediately after its creation the electron and the photohole interact via an unscreened Coulomb interaction. The interaction is unscreened because the system of the valence electrons needs a finite time to react to the existence of the photohole $[\epsilon(t=0)=1]$. So if the hole is sufficiently localized-as are, e.g., holes in the $d$ bands of the noble metals - the potential will lead to bound states when plugged into the Schrödinger equation. We will show later in this paper why localization is important. In the case of extreme localization, a point charge, the eigenvalues will form a Rydberg series as in a hydrogen atom. With progressing time the initially bare potential becomes more and more screened by the valence electrons until at large times the total potential-bare potential plus screening potential-reaches its asymptotic shape. Whereas the bare potential typically has bound states this is in metals no longer the case for the total potential at large times. Considering again the simple case of a point charge in a homogeneous electron gas, the total potential will be the Thomas-Fermi potential $v_{\text {tot }}(t$ $\rightarrow \infty)=v_{\mathrm{TF}}=\left(e^{2} / r\right) \exp \left\{k_{\mathrm{TF}} r\right\}$, where $k_{\mathrm{TF}}$ is the ThomasFermi wave vector. ${ }^{13}$ For the electronic densities which occur in nature-described by a Wigner-Seitz radius of $2 \leqslant r_{s}$ $\leqslant 5-v_{\mathrm{TF}}$ has no bound states. This is the reason why there are no excitons in metals on a long time scale, i.e., under stationary conditions. In semiconductors and insulators, on the other hand, the resulting total potential for $t \rightarrow \infty$ will be a Coulomb potential screened by the (frequency independent) dielectric constant $\epsilon, v_{\text {tot }}(t \rightarrow \infty)=v_{\mathrm{c}}=e^{2} /(\epsilon r)$. For typical values of $\epsilon$ in semiconductors this potential leads to bound states which are observed in experiments as excitons.

This discussion makes clear that even in metals excitonic states are possible. However, they can only occur on a very short time scale. We therefore call them transient excitonic states. But this short time scale is precisely what is probed in TR-2PPE spectroscopy using ultrashort laser pulses. Therefore it was not before the advent of lasers with the necessary very short pulses that transient excitonic states in metals could have been detected. Note that the process described 
above is very general. Consequently, transient excitons can be expected in a variety of metals.

In this paper we will briefly review the model introduced in Ref. 11 and we discuss the differences and the common features of the three noble metals and $\mathrm{Al}$ as an example of a nearly free electron (NFE) metal with respect to the transient excitonic states.

Let us start by summarizing the model presented in Ref. 11. Our starting point is a localized hole in the ( $d$ bands of the) valence bands $n^{h}(\mathbf{r})$. The hole is taken as the negative of the electronic charge distribution of a localized electron. Using Poisson's equation, $n^{h}(\mathbf{r})$ can be related to a potential which we treat as an external potential switched on at $t=0$, the time when the electron-hole pair is created. The dominating spherical part is given by ${ }^{11}$

$$
v_{\text {ext }}(r, t)=4 \pi e \int_{0}^{\infty} d r^{\prime} r^{\prime 2} \frac{1}{r_{>}} n^{h}\left(r^{\prime}\right) \Theta(t) .
$$

Here $r_{>}$denotes the larger of $r$ and $r^{\prime}$, respectively. $\Theta(t)$ is the usual step function and $n^{h}(r)$ is the spherical part of $n^{h}(\mathbf{r})$. We now ask how the valence electrons react to the perturbation set up by $v_{\text {ext }}(r, t)$ and answer this question within linear response theory, i.e., we calculate the induced density

$$
n_{\text {ind }}(\mathbf{r}, t)=\int d^{3} r^{\prime} \int_{-\infty}^{\infty} d t^{\prime} \chi\left(\mathbf{r}, \mathbf{r}^{\prime} ; t-t^{\prime}\right) v_{\mathrm{ext}}\left(\mathbf{r}^{\prime}, t^{\prime}\right)
$$

where $\chi\left(\mathbf{r}, \mathbf{r}^{\prime} ; t-t^{\prime}\right)$ is the (retarded) density-response function. It is calculated in Fourier space from the polarizability $P$ by solving the usual matrix equation. ${ }^{11,14} P$ is calculated using the familiar expression. ${ }^{11,14}$ In order to evaluate $P$ we use $29 \mathbf{k}$ points in the irreducible wedge of the Brillouin zone and 200 bands, i.e., we consider transitions up to approximately $300 \mathrm{eV}$ above the Fermi energy. This means that even for large wave vectors the polarizability is converged with respect to the number of bands considered. To solve the matrix equation for the density-response function we use two complete shells of reciprocal lattice vectors. Once the induced density has been obtained it can be related to an induced potential $v_{\text {ind }}(r, t)$ via Poisson's equation. The timedependent total potential is finally given as

$$
v_{\text {tot }}(r, t)=v_{\text {ext }}(r, t)+v_{\text {ind }}(r, t) .
$$

Now optical excitations are vertical transitions from an initial to a final state. In the case of excitonic excitations the energy level of the final states is shifted downwards because of the mutual interaction between the electron and the hole. According to this process we evaluate the energies of the transient excitonic states for given, fixed times $t$ as follows. For a given wave vector, say, in the direction $\Gamma-L$, the effective mass is calculated for the initial and the final band. For the noble metals we used one of the flat $d$ bands as initial state and the lowest lying $s p$-like band above the Fermi energy as final band (the thick solid lines in Fig. 1). From the effective masses of these two bands we obtain the reduced mass $\mu$ which is used in the Schrödinger equation. The Schrödinger equation is solved for the potentials calculated
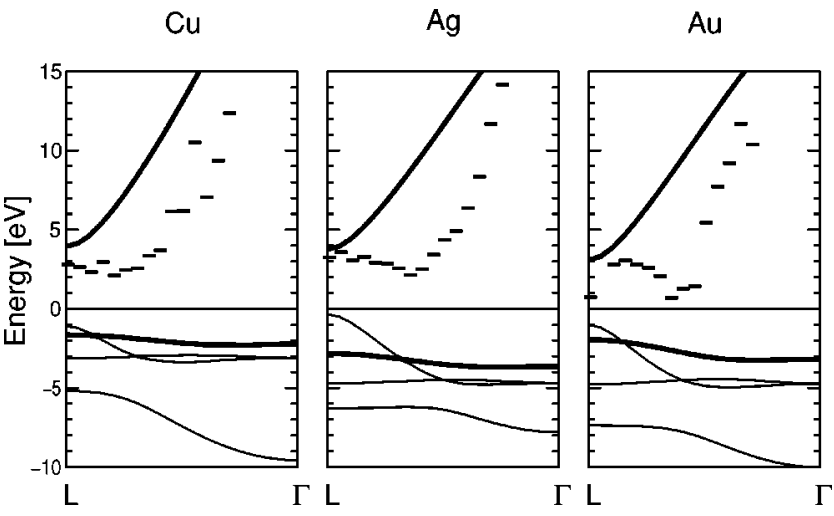

FIG. 1. Comparison of transient excitonic states (horizontal bars) in $\mathrm{Cu}, \mathrm{Ag}$, and $\mathrm{Au}$ calculated for the $L-\Gamma$ panel of the band structure [(100) direction]. Due to their rather similar band structure the three noble metals reveal comparable transient excitonic states. The main difference in the band structures is the energetic position of the $d$ bands. They are 1-2 eV deeper in $\mathrm{Ag}$ than they are in $\mathrm{Cu}$ or $\mathrm{Au}$. This is the reason why transient excitonic states have not yet been detected with the pump photon energies typically used in the experiments. The bands which are denoted by thick solid lines were used in the calculation as initial and final states of the optical transition [see the discussion following Eq. (3)].

via Eq. (3) for fixed times $t$. The resulting lowest eigenvalue is plotted with respect to the final band energy. This leads to the energy levels denoted as horizontal bars in Fig. 1.

Figure 1 shows the transient excitonic states for $\mathrm{Cu}, \mathrm{Ag}$, and $\mathrm{Au}$ in the $\Gamma-L$ direction plotted into the band structure of the respective elements. The potentials which were used when solving the Schrödinger equation correspond to $t$ $=0.01$ fs. Since the band structure of these three elements are rather similar one can expect that the positions of the transient excitonic states will fall in a comparable energy region. This is exactly what is shown by Fig. 1. All of the three elements reveal comparable transient excitonic states. This is the desired result for $\mathrm{Cu}$ and for $\mathrm{Au}$, where the calculated energetic positions are in the region in which the experiments detected these states. For Ag, however, experiments have not yet found states in the $\Gamma-L$ direction, contrary to the results of our calculations. The resolution of this seeming contradiction is quite simple. The $d$ bands in Ag are about 1 to $2 \mathrm{eV}$ deeper than the $d$ bands in $\mathrm{Cu}$ and $\mathrm{Au}$. With the pump photon energies used in the experiments these states can just not be detected. Using photons with higher energy these states should be seen.

The situation shown in Fig. 1 is just a snapshot at a given time. Due to the dynamic character of the total potential the corresponding eigenvalues are functions of time. Especially, they are oscillating because $v_{\text {tot }}$ is a damped oscillating function with respect to $t .{ }^{11}$

Another element where transient excitonic states also have not been detected is Al. The measured lifetimes of excited electrons in $\mathrm{Al}$ can be explained as the lifetime of single-particle states in the $W-L, W-X$, and $W-K$ directions, respectively. ${ }^{7}$ These states can easily be reached by vertical transitions with the photon energies used in the experiments. The calculated lifetimes of these states within the $G W$ ap- 


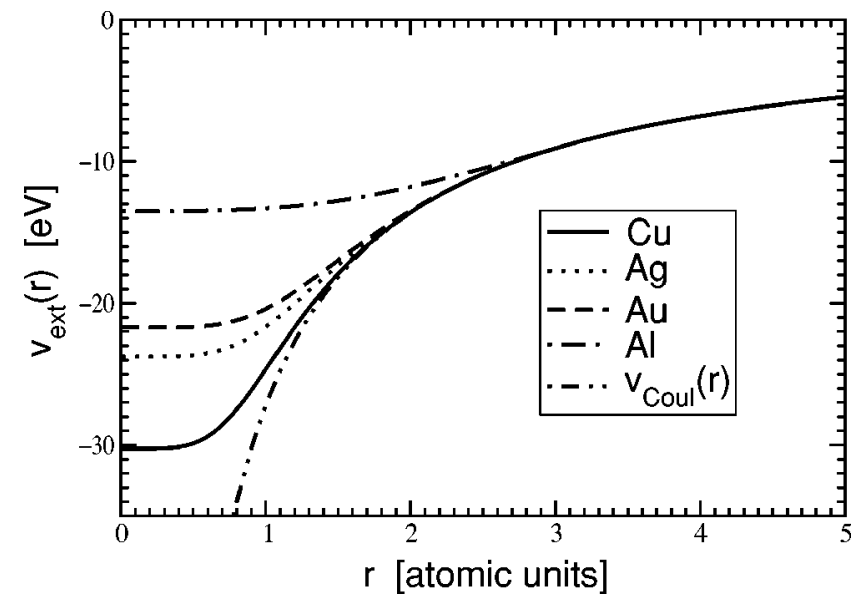

FIG. 2. The external potentials due to a hole in $\mathrm{Cu}$ (solid line) and $\mathrm{Al}$ (dot-dashed line), respectively. The double-dotted-dashed line denotes the Coulomb potential. Due to the delocalized character of the electrons in $\mathrm{Al}$ the potential is very shallow and does therefore not lead to bound states. The potential in the case of $\mathrm{Cu}$ is much deeper. Even during the build up of the screening it leads to bound states. The dotted and dashed line show the external potentials of $\mathrm{Ag}$ and $\mathrm{Au}$, respectively.

proximation agree well with the experimental data. Since our model is based on the assumption of a localized hole it is quite obvious that $\mathrm{Al}$ - which in many respects can be treated as a nearly free electron metal-should not build up transient excitonic states. This conspicuous though qualitative argument can be quantified by comparing the external potentials of a localized hole in a $\mathrm{Cu} d$ band and hole in the valence bands of $\mathrm{Al}$, respectively. Figure 2 shows the two potentials. Whereas the potential in $\mathrm{Cu}$ is rather deep the potential in $\mathrm{Al}$ is very shallow. This is a consequence of the delocalized character of the electrons or holes in Al. Because of the flatness not even the external potential in Al leads to bound states not to talk of the screened potential.
Note that our calculations only give a rough estimate for the time during which the electron has to be trapped, i.e., the time during which the excitonic state has to be formed. Once the exciton has been formed the physical situation is different. This has to be considered in a calculation of the lifetime of these states.

To summarize, we applied our model for transient excitonic states to the noble metals $\mathrm{Ag}$ and $\mathrm{Au}$. We showed that in both elements transient excitonic states do exist. The reason why they have been detected so far in Au but not in $\mathrm{Ag}$ is only due to the different excitation energies needed to excite the transient excitons in the two elements. We predict that transient excitonic states in Ag do exist and that they will be detected once higher energies for the pump photons will be used. We also discussed the case of $\mathrm{Al}$ as a prototype for a delocalized electron system and showed why these kind of systems cannot build up transient excitonic states. These calculations give further evidence that our model is consistent and valid and they therefore also show once more that transient excitonic states in metals do exist and are detected in experiments using TR-2PPE spectroscopy.

Since the existence of transient excitonic states is now evident the natural next step is necessary, namely, the calculation of the lifetime of electrons trapped in transient excitonic states by solving the full Bethe-Salpeter equation using the full time-dependent interaction, i.e., going beyond the static approximation done so far. The determination of the lifetime is beyond the scope of our simple model. This is a formidable task and work in this direction is under way. Solving the Bethe-Salpeter equation will also give more exact numbers for the energetic position of these states and the time during which electrons can be captured in these states.

G. Ertl is gratefully acknowledged for his interest and generous support. We thank R. Keyling for enlightening discussions. This work is supported by the Deutsche Forschungsgemeinschaft through SFB 450.
${ }^{1}$ S. Ogawa, H. Nagano, and H. Petek, Phys. Rev. B 55, 10869 (1997).

${ }^{2}$ E. Knoesel, A. Hotzel, and M. Wolf, Phys. Rev. B 57, 12812 (1998).

${ }^{3}$ M. Bauer, S. Pawlik, and M. Aeschlimann, Proc. SPIE 3272, 201 (1998).

${ }^{4}$ J. Cao, Y. Gao, R.J.D. Miller, H.E. Elsayed-Ali, and D.A. Mantell, Phys. Rev. B 56, 1099 (1997).

${ }^{5}$ J. Cao, Y. Gao, H.E. Elsayed-Ali, R.J.D. Miller, and D.A. Mantell, Phys. Rev. B 58, 10948 (1998).

${ }^{6}$ Zahlenwerte und Funktionen aus Naturwissenschaften und Technik, Landolt-Börnstein, New Series, Group III, Vol. 17, Pt. a (Springer, New York, 1982).
${ }^{7}$ W.-D. Schöne, R. Keyling, M. Bandić, and W. Ekardt, Phys. Rev. B 60, 8616 (1999).

${ }^{8}$ R. Keyling, W.-D. Schöne, and W. Ekardt, Phys. Rev. B 61, 1670 (2000).

${ }^{9}$ I. Campillo, J.M. Pitarke, A. Rubio, E. Zarate, and P.M. Echenique, Phys. Rev. Lett. 83, 2230 (1999).

${ }^{10}$ F. Aryasetiawan and O. Gunnarsson, Rep. Prog. Phys. 61, 237 (1998).

${ }^{11}$ W.-D. Schöne and W. Ekardt, Phys. Rev. B 62, 13464 (2000).

${ }^{12}$ Here we neglect the tensor properties of the dielectric function.

${ }^{13}$ G. D. Mahan, Many-Particle Physics (Plenum, New York, 1990).

${ }^{14}$ A.G. Eguiluz, A. Fleszar, and J.A. Gaspar, Nucl. Instrum. Methods Phys. Res. B 96, 550 (1995). 